\title{
Modified Ertel's Potential Vorticity as a Climate Variable
}

\author{
M. V. KURGANSKY \\ A. M. Obukhov Institute of Atmospheric Physics, Russian Academy of Sciences, Moscow, Russia
}

\section{A. PISNICHENKO*}

Center for Weather Forecast and Climate Investigations/National Institute of Space Research (CPTEC/INPE), Cachoeira Paulista, Sao Paulo, Brazil

(Manuscript received 18 May 1998, in final form 17 May 1999)

\begin{abstract}
Fundamental freedom existing in Ertel's general definition of potential vorticity (PV) is used to construct an essential invariant measure in $(q, \chi)$ space, where $q$ is an "optimal" modified PV and $\chi$ is a monotonic increasing function of potential temperature, which enters $q$. This novel measure is preserved in idealized climate processes, when external diabatic heating and frictional forcing, being applied to zonally oriented infinitely thin $(q, \chi)$ tubes (formed at the expense of intersection between isoscalar surfaces $q=$ constant and $\chi=$ constant), annihilate each other. A reference stationary airmass distribution on $(q, \chi)$ values is introduced for both hemispheres, separately, which corresponds to a hypothetical atmospheric climate equilibrium state. This reference distribution, with no dependence on $\chi$, is characterized by exponentially decaying function of the absolute value of $q$ and has the same total amount of "PV substance" (atmospheric vorticity charge) as the actual atmospheric state. Comparison of the actual monthly mean airmass distribution on $q$ (calculated on the basis of 1980-89 European Centre for Medium-Range Weather Forecasts data) with this reference stationary distribution by their informational entropy values enables one to quantify a degree of nonequilibrium of real atmospheric climate processes, with respect to the introduced invariant measure.
\end{abstract}

\section{Introduction}

Ertel's potential vorticity (PV) plays a central role in modern dynamical meteorology, including theoretical investigations, as well as applications to the modeling and diagnosis of tropospheric and, of importance now, largescale stratospheric processes. There are review papers by Hoskins et al. (1985), Kurgansky and Tatarskaya (1987), and Holton et al. (1995) specially devoted to these broad topics. Not so frequently, potential vorticity is considered as an indicator of atmospheric climate processes. Until now, its use in the climate theory studies has been focused mainly on the analysis of time-mean PV-flux calculations (e.g., Koshyk and McFarlane 1996).

Both the well-known freedom available in the definition of potential vorticity (Ertel 1942; Haynes and McIntyre 1990; Lait 1994) and the theorem on conservation of vor-

\footnotetext{
* Permanent affiliation: A. M. Obukhov Institute of Atmospheric Physics, Russian Academy of Sciences, Moscow, Russia.

Corresponding author address: Dr. Igor A. Pisnichenko, Universidade Federal do Paraná, Departamento de Fisica, Caixa Postal 19081, CEP: 81531-990, Curitiba, Paraná, Brazil.

E-mail: igor@ fisica.ufpr.br
}

ticity charge (PV substance) in a compressible stratified fluid (Obukhov 1962; Haynes and McIntyre 1990) give us a foundation for construction of a new PV-related functional that is quasi-conservative under diabatic and frictional factor influence, and that is used for a rational description of slowly varying climate processes. This could serve as a basis for applying statistical principles, exploiting PV thinking, to climate investigations.

Obukhov (1964) has shown that for adiabatic motion an invariant $\mu(\Omega, \Theta)$ exists $[\Omega$ is the potential vorticity, $\Theta$ is the potential temperature (PT)], which represents the atmospheric airmass portion enclosed within a tube formed by the intersection of isoscalar surfaces $\Theta, \Theta+d \Theta=$ constant and $\Omega, \Omega+d \Omega=$ constant. He pointed out that $\mu(\Omega, \Theta)$ may be treated statistically as a probability density of $\Omega$ and $\Theta$ values for an arbitrarily chosen air parcel. In this paper, we intend to show that it is possible to find such a transformation, $\mathbf{T}:=($ Ertel's $\mathrm{PV}, \Theta) \Rightarrow$ [modified $\mathrm{PV} \equiv \mathrm{MPV}, \chi(\Theta)]$, which results in the constancy of air mass $\mu$ enclosed within an infinitely thin (MPV, $\chi$ )-tube, for diabatic and frictional forcing of a special kind. This gives us the right to assume that a time-invariant measure in (MPV, $\chi$ ) space exists. The existence of such a measure allows us to introduce the probability density $\mu(\mathrm{MPV}, \chi)$ for description of realistic diabatically and frictionally driven climate processes in the terrestrial atmosphere. A 
visualization of a particular transformation $\mathbf{T}$, used in the paper, is given in Fig. 1. A detailed description of the procedure for finding the transformation $\mathbf{T}$ is proposed in sections 2 and 3.

Globally, the atmosphere is permanently observed in the state of chaos, which serves as a background for individually better-distinguishable irregular processes of different temporal and spatial scales (the so-called atmospheric circulation regimes). Chaotic dynamics of the atmospheric climate system occurring on its attractor (Lorenz 1995) could be regarded as quasi equilibrium in that sense, that on the attractor we assume [and for certain atmospheric climate models it could be strictly proved (Dymnikov and Filatov 1997)] the existence of an unique stationary statistical distribution; that is, an essential invariant measure on the attractor does exist. To characterize the attractor of the atmospheric climate system in (MPV, $\chi$ )-terms we propose the use of a hypothetical reference state, which the atmosphere closely approaches but never reaches precisely. It will be shown that this reference state is described by a probability density $\mu$ (which is defined for two hemispheres, separately) depending on MPV only. In this paper, the reference state will be introduced somewhat ad hoc, as "a reasonable first guess," under the sole imposed condition that it has the maximum of informational entropy, provided that the total amount of MPV substance-the atmospheric vorticity charge-over a hemisphere is a prescribed constant quantity. Justifications for this axiomatic approach are (i) the lack of internal contradictions inside the theoretical (speculative) scheme used and (ii) the agreement between theoretical predictions and observational evidence. The reference $\mu(\mathrm{MPV})$ distribution describes a "climate background noise" and corresponds to the regime of complete statistical equilibrium of the atmosphere. The actual atmospheric climate system is not statistically equilibrium, by all means, but the very existence of a stationary statistical distribution $\mu$ (MPV) for the climate system attractor and observed closeness between the actual distribution and this reference $\mu$ distribution makes this approach appropriate.

A comprehensive characterization of an airmass statistical distribution on MPV and $\chi$ (or EPV and $\Theta$ ) is its informational entropy $H$ defined according to Shannon and von Neumann (e.g. Katz, 1967). The entropy $H$ can be interpreted as a general measure of the degree of uncertainty in MPV and $\chi$ values for a randomly chosen air parcel. The corresponding informational entropy deficit (the difference between $H$ values for reference and actual airmass statistical distributions) tends to a minimum, when (MPV, $\chi$ )-coordinates are used for a description of real atmosphere. The quasi-permanent external diabatic and frictional forcing is balanced in such a way that the air mass contained in (MPV, $\chi$ ) tubes is nearly constant. The small unbalanced part of external thermal forcing (e.g., due to nonuniform solar heating of the earth's surface, and seasonal course in insolation) tends to turn the atmosphere away from the reference state; but the internal dynamical processes, with the characteristic timescale of about one week, do not permit the atmosphere to diverge from it significantly. As a result, the monthly mean airmass statistical distribution on MPV and $\chi$ is perpetually observed in the vicinity of the reference $\mu(\mathrm{MPV})$ distribution.

In section 2 of this paper an arbitrariness in Ertel's potential vorticity definition is discussed and used in such a way as to arrive at MPV concept, which corresponds to airmass distribution on MPV having a finite first momentum (the atmospheric vorticity charge). A justification of the transformation $\mathbf{T}$ to new (MPV, $\chi$ )coordinates is represented in section 3. A computational method for the determination of these coordinates, which is based on searching for the minimum of informational entropy difference between actual and reference distribution, is proposed. An algorithm for the construction of the discrete version of the reference distribution as well as of its informational entropy estimation is given in the appendix. In section 4 , the efficiency of this method is demonstrated using 1980-89 data from the European Centre for Medium-Range Weather Forecasts (ECMWF). Possible applications of our approach to the problem of atmospheric climate variability diagnosis are discussed briefly.

\section{Modified Ertel's potential vorticity}

Ertel's (1942) vorticity theorem states that a quantity constructed by multiplying the specific volume $\rho^{-1}(\rho$ is the density) by the scalar product of the absolute vorticity $\mathbf{Z}$ and the gradient of a materially conserved scalar quantity $\Psi(p, \rho)$ ( $p$ is the pressure) is constant in an individual fluid parcel if (i) a fluid is inviscid and (ii) only external potential forces are present. An important special case occurs when $\Psi$ coincides with the potential temperature $\Theta$, as it was originally proposed by Ertel (1942). Under adiabatic approximation one has

$$
\frac{D}{D t} \frac{\mathbf{Z} \cdot \nabla \Theta}{\rho}=0,
$$

where $D / D t$ denotes the material time derivative. Commonly, (1) is referred to as Ertel's potential vorticity conservation law. As it is widely known (e.g., Haynes and McIntyre 1990; Lait 1994), one can replace $\Theta$ in (1) with its arbitrary differentiable monotonic function $\chi(\Theta)$. We restrict ourselves to $\chi$ functions, obeying two additional conditions: (i) $d \chi / d \Theta>0$ and (ii) $\chi \Rightarrow 0$ when $\Theta \Rightarrow \infty$. As a consequence, $\chi$ is negative everywhere. Following Obukhov (1964) we shall take $\chi(\Theta)$ $=-p^{*}(\Theta) / g$, where $p^{*}$ is a reference pressure dependent upon $\Theta$ and $g$ is the gravity acceleration. Hereafter, we apply the term "potential vorticity" to the quantity

$$
q=\rho^{-1} \mathbf{Z} \cdot \boldsymbol{\nabla}\left[-p^{*}(\Theta) / g\right],
$$

[reserving the name "modified potential vorticity" for $q$ with a special choice of $p^{*}(\Theta)$ ] having an order of the Coriolis parameter magnitude $\left(10^{-4} \mathrm{~s}^{-1}\right)$. In this 

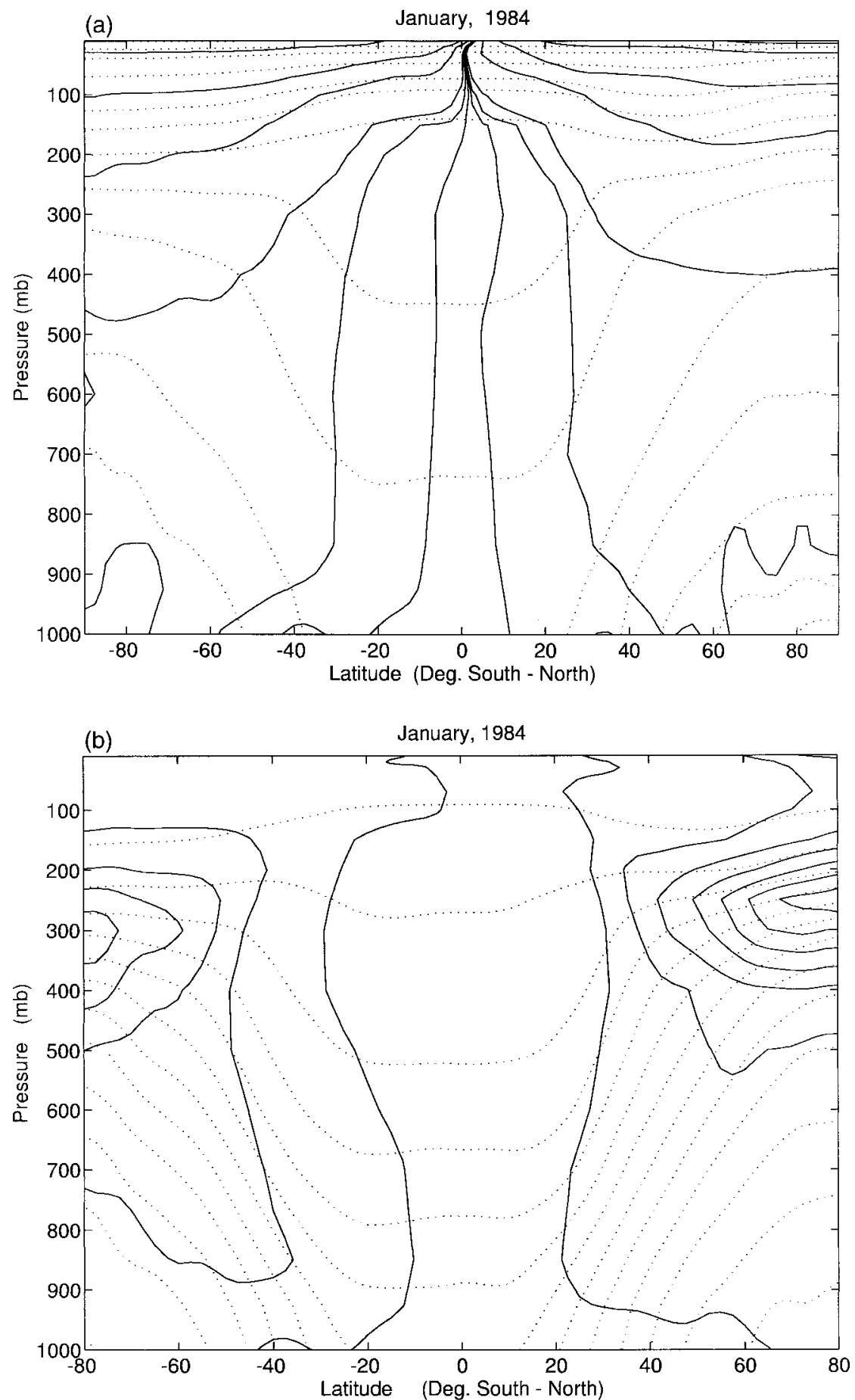

FIG. 1. Zonally averaged latitude-height cross section of (a) Ertel's potential vorticity (solid line: contours are in units of $10^{-6} \mathrm{~K} \mathrm{~m}^{2} \mathrm{~kg}^{-1} \mathrm{~s}^{-1}$ and display values of $-4000,-1000,-400$, $-100,-40,-6,-2,0,2,6,40,100,400,1000,4000)$ and potential temperature (dotted line: contours display the values of 250, 260, 270, 280, 290, 300, 310, 330, 350, 380, 420, 500, 600, 800), (b) MPV (solid line: interval between contours equals to $1.04 \mathrm{~s}^{-1}$; extreme left contour has a value $-5.51 \times 10^{-4} \mathrm{~s}^{-1}$ and extreme right contour has a value $6.96 \times 10^{-4} \mathrm{~s}^{-1}$ ) and $\chi$ function (dotted line: interval between contours equals to $1.22 \cdot 10^{3} \mathrm{~kg} \mathrm{~m}^{-2}$ and upper contour has a value $-1.44 \times 10^{3} \mathrm{~kg} \mathrm{~m}^{-2}$; values of contours increase to the direction of the earth's surface). 
case, the potential vorticity becomes equal to the absolute vorticity component, which is orthogonal to isentropic surfaces and has been reduced to standard vertical stratification conditions. Along with the Coriolis parameter, the potential vorticity $q$ mainly varies in the meridional direction, while $\Theta$ changes predominately in altitude. Therefore, the family of isoscalar surfaces $q=$ const and $\Theta=$ const may be regarded as a natural coordinate system, advantageous for displaying largescale atmospheric circulation patterns (e.g., Kurgansky and Tatarskaya 1987; Lait 1994; Pisnitchenko and Kurgansky 1996). In this paper we intend to show how a transition to new (MPV, $\chi$ )-coordinates gives additional possibilities for description of climate processes. One can clearly see the difference between (EPV, $\theta$ )- and (MPV, $\chi$ )-coordinates in Fig. 1. We would like to draw attention to the fact that both MPV and $\chi$ isolines are nearly equidistant (in the coordinates used), but the intervals between Ertel's PV and PT isopleths are exponentially increasing, approximately. In their potential vorticity computations, Kurgansky and Tatarskaya (1987), basing on the analysis of real data, proposed the use of the function

$$
p^{*}=A-B \operatorname{arctg}\left[C\left(\Theta-\Theta_{0}\right)\right] \text {, }
$$

where $A=681 \mathrm{hPa}, B=433.5 \mathrm{hPa}, C=0.04614 \mathrm{~K}^{-1}$, and $\Theta_{0}=283 \mathrm{~K}$ are the constants, as a fairly good approximation to the climatological $p^{*}(\Theta)$ profile.

From (2) it follows that the integral

$$
Z_{\mathrm{A}}=\iiint_{V} q \rho d V \equiv \iiint_{V}\left[\mathbf{Z} \cdot \nabla\left(-p^{*}(\Theta) / g\right)\right] d V,
$$

taken over the entire atmosphere volume $V$, becomes a well-defined finite quantity. Its precise value is not sensitive to the exact form of atmospheric outer boundary provided that this boundary is placed at a sufficient height to enclose practically all the atmospheric mass. Here, $d V$ is the volume element. Following Obukhov (1962) and Kurgansky (1991), $Z_{A}$ is named the "atmospheric vorticity charge," which stresses an analogy with the electric charge conservation law in electrodynamics. Bishop and Thorpe (1994) and Thorpe and Bishop (1995) have also commented on the interpretation of potential vorticity in terms of electrostatic charge. When $V$ contains the hemispheric portion of the atmosphere one arrives at the atmospheric vorticity charge either over the Northern Hemisphere $Z_{\mathrm{NH}}$ or over the Southern Hemisphere $Z_{\mathrm{SH}}$. The net atmospheric vorticity charge is equal to the sum of the positive contribution from the Northern Hemisphere and the negative one from the Southern Hemisphere: $Z_{A}=Z_{\mathrm{NH}}+Z_{\mathrm{SH}}$. The resultant quantity is close to zero, if annually averaged, but still may acquire very small negative values, thus showing thermal asymmetry between the hemispheres (Kurgansky 1991).

\section{3. "Climatological" potential vorticity definition}

\section{a. Dynamical fundamentals}

Under the influence of diabatic heating $\dot{\Theta}$ and frictional forces $\mathbf{F}$ the potential vorticity $q$ transforms according to the equation (e.g., Haynes and McIntyre 1990)

$$
\dot{q}=\rho^{-1}[\mathbf{Z} \cdot \boldsymbol{\nabla} \dot{\chi}+\nabla \chi \cdot \nabla \times \mathbf{F}],
$$

where $\chi(\Theta)=-p^{*}(\Theta) / g$, and a dot above variables denotes the material time derivative $D / D t$. Equation (4) does not change its form under the transformation $\chi \Rightarrow$ $\chi^{*}=\Phi(\chi), q \Rightarrow q^{*}=\Phi^{\prime} q$. Here, $\Phi$ is an arbitrary differentiable function and $\Phi^{\prime}$ represents a derivative of $\Phi$ with respect to the argument $\chi$.

The isoscalar surfaces $q=$ constant and $\chi=$ constant divide the atmosphere into $(q, \chi)$-solenoids (tubes), along which air parcels flow during adiabatic and frictionless processes. The corresponding solenoidal vector $\mathbf{A}=\boldsymbol{\nabla} q \times \boldsymbol{\nabla} \chi$ obeys the equation (Kurgansky and Tatarskaya 1987)

$$
\frac{D}{D t} \frac{\mathbf{A}}{\rho}-\left(\frac{\mathbf{A}}{\rho} \cdot \nabla\right) \mathbf{v}=\frac{1}{\rho} \nabla \times(\dot{q} \nabla \chi-\dot{\chi} \nabla q),
$$

which readily follows from Ertel's commutative formula (e.g., Hollmann 1964). Friedmann (1934) proved a theorem, which generalizes the well-known Helmholtz vorticity theorems. It states that the necessary and sufficient condition for the conservation of both vectorial filaments and vectorial tubes of an arbitrary vector field a is the fulfilling of the equation

$$
\frac{D}{D t} \frac{\mathbf{a}}{\rho}-\left(\frac{\mathbf{a}}{\rho} \cdot \nabla\right) \mathbf{v}=0 .
$$

The term "conservation" means that (i) both a filaments and $\mathbf{a}$ tubes are frozen in a fluid, that is, move along with fluid parcels; and (ii) the strength of a tubes, that is, the flux of the vector a across tubes' cross sections, is time constant. For atmospheric zonal circulation state, conservation of the vector A in Friedmann's (1934) sense is exactly equivalent to preservation of air mass, enclosed within an infinitely thin $(q, \chi)$ solenoid.

The vector $\mathbf{A}$ is conserved not only in an adiabatic and frictionless case, when $\dot{q}=\dot{\chi}=0$, but also under a less restrictive assumption of

$$
\dot{q} \nabla \chi-\dot{\chi} \nabla q=-\nabla B
$$

where $B$ is a scalar function of position vector $\mathbf{x}$ and time $t$. $^{1}$

\footnotetext{
${ }^{1}$ A rigorous formulation of mass continuity equation in $(q, \chi)$ coordinates is readily obtained when following literally the arguments given in Nakamura (1995). For a particular class of quasi-zonal $(q, \chi)$ tubes, with $\dot{q}=\dot{q}(q, \chi, t)$ and $\dot{\chi}=\dot{\chi}(q, \chi, t)$, that is, when these variables depend on a lengthwise (longitudinal) coordinate implicitly, via $q$ and $\chi$, condition (5) is equivalent to preservation of air mass, enclosed within an infinitely thin $(q, \chi)$-solenoid. The case of strictly zonal atmospheric circulation, mentioned in the main text, enters here as a special case.
} 
In order for (5) to hold for variables $\left[q^{*}=f(\chi) q, \chi^{*}\right.$ $=h(\chi)$ ], with a certain new function $B^{*}$, the transformation $q^{*}=f(\chi) q, \chi^{*}=h(\chi), f=n^{-2} / h^{\prime}$ has to be performed, with the Jacobian determinant $\partial\left(q^{*}, \chi^{*}\right) /$ $\partial(q, \chi)=n^{-2}$. Here, $f$ and $h$ are arbitrary differentiable functions, and $n$ is a numerical constant. This is consistent with the above-written demand for preservation of a covariant form of (4) in the case of $\left[\Phi^{\prime}\right]^{2}=\left[h^{\prime}\right]^{2}$ $=n^{2}$, that is, when $\chi^{*}= \pm \chi / n$.

Formally, based on Lorenz's (1955) ideas, one may suppose the existence of a weightless perfect gas underground. Here, all isentropic surfaces could be regarded as closed surfaces, with PT values covering a range of $\Theta_{\min } \leq \Theta<\infty$, where $\Theta_{\min }$ is the minimum PT value in the atmosphere. The entire atmospheric mass lies well above the isentropic surface $\Theta_{\min }=$ const. So, by $\chi$ definition as a pressurelike variable, it is natural to assume that $\chi^{*}\left(\Theta_{\min }\right)=\chi\left(\Theta_{\min }\right)$. Consequently, $n=$ \pm 1 , which shows that $\chi^{*} \equiv \chi$. We conclude, that Eqs. (4) and (5) could be fulfilled simultaneously, if at all, for a single choice of $\chi$. This choice corresponds to the case of an "optimal" potential vorticity modification.

It should be stressed that our speculations do not prove the existence of this preferred choice of $\chi$. What do we prove is that this choice is unique, if possible at all. In the subsequent sections an approximate computational method for the optimal $\chi$ function determination will be developed.

\section{b. Basic statistical arguments}

Let us consider the quantity $\mu(q, \chi) d q d \chi$ equal to the part of the total atmospheric mass, enclosed within a solenoid formed by intersection between the isoscalar surfaces $q, q+d q=$ constant and $\chi, \chi+d \chi=$ constant. If we allow that $\iint \mu(q, \chi) d q d \chi=1$, where integration is extended over all $q$ and $\chi$ values, then $\mu(q, \chi)$ may be regarded as the probability density of $q$ and $\chi$ values for a randomly chosen air parcel (Obukhov 1964). By definition, the atmospheric vorticity charge equals to $Z_{A}$ $=m_{\mathrm{A}} Q_{\mathrm{A}}$, where $m_{\mathrm{A}}$ is the total atmospheric mass, and $Q_{\mathrm{A}}=\iint q \mu(q, \chi) d q d \chi$ is the first moment of $\mu(q, \chi)$ distribution. The normalized-by-unity $\mu(q, \chi)$ distributions were calculated separately for the Northern Hemisphere (NH) and the Southern Hemisphere (SH). They are $\mu_{\mathrm{NH}}(q, \chi)$ and $\mu_{\mathrm{SH}}(q, \chi)$, respectively. A remarkable piece of empirical evidence was used that nearly all air parcels with positive $q$ are confined in the $\mathrm{NH}$ and nearly all parcels with negative $q$ in the $\mathrm{SH}$, correspondingly. This holds with accuracy better than $0.4 \%$, if the corresponding airmass portion is estimated (Kurgansky 1991). This makes $\mu_{\mathrm{NH}}(q, \chi)$ and $\mu_{\mathrm{SH}}(q, \chi)$ statistically independent. Because the total atmospheric mass is equipartitioned between the hemispheres (actually, it happens with very good accuracy), it is taken that $\mu(q, \chi)=0.5 \mu_{\mathrm{NH}}(q, \chi)+0.5 \mu_{\mathrm{SH}}(q, \chi)$. The characteristic $2 Q_{\mathrm{A}}$ is thus equal to the sum of the positive contribution from the $\mathrm{NH} Q_{\mathrm{NH}}=\int q \mu_{\mathrm{NH}}(q, \chi) d q d \chi$ and the negative one from the SH $Q_{\mathrm{SH}}=\int q \mu_{\mathrm{SH}}(q, \chi) d q$ $d \chi$. Later on, the indices "NH" and "SH" will be dropped out. It is assumed that all the following theoretical results are equally valid for each hemisphere, with account for the $q$ sign.

\section{c. Reference distribution}

We seek a stationary reference airmass distribution $\mu$ between infinitely thin, quasi-zonally oriented $(q, \chi)$ solenoids, when the condition (5) for their mass preservation is satisfied and a corresponding invariant measure in $(q, \chi)$-functional space is specified. Following classic arguments by Gibbs (1948), leading to his canonical distribution, and taking into account the principle of "modified potential vorticity substance" conservation, the most simple and natural choice is to set $\mu(q, \chi)=$ $\mu_{\mathrm{B}}(q, \chi)=\mu_{0} \exp (-\alpha q)$, where $\mu_{0}$ and $\alpha$ are positive constants. Without loss of generality, the Northern Hemisphere case, when $q>0$, is considered. Because of the first-moment $Q$ definition, this immediately leads to the distribution

$$
\mu_{\mathrm{B}}(q, \chi)=\mu_{0} \exp (-q / Q),
$$

with $Q=1 / \alpha$. The distribution (6) has two important properties, which might serve as the guiding principles in the course of its derivation: (i) equipartitioning of air mass between equal intervals $d \chi$; and (ii) multiplication of corresponding probability densities when arbitrarily splitting $q$ onto a sum of two items $q_{1}$ and $q_{2}\left[\mu_{\mathrm{B}}\left(q_{1}+\right.\right.$ $\left.\left.q_{2}, \chi\right)=\mu_{\mathrm{B}}\left(q_{1}, \chi\right) \mu_{\mathrm{B}}\left(q_{2}, \chi\right)\right]$.

Integration of $\mu_{\mathrm{B}}(q, \chi)$ over all $q$ gives $\int \mu_{0} Q d \chi=$ 1 (here, we use the normalization condition), and, as a consequence, ${ }^{2}$

$$
\begin{aligned}
\mu_{\mathrm{B}}(q) & =\int_{Q^{-1} \exp (-q / Q) .} \mu_{\mathrm{B}}(q, \chi) d \chi=\exp (-q / Q) \int \mu_{0} d \chi \\
& \left.={ }^{2}\right)
\end{aligned}
$$

In $(q, \Theta)$-coordinates used in Kurgansky and Tatarskaya (1987) and Obukhov et al. (1988), one has

$$
\begin{aligned}
\mu_{\mathrm{B}}(q, \Theta) & =|\partial(q, \chi) / \partial(q, \Theta)| \mu_{\mathrm{B}}(q, \chi) \\
& =|\partial(q, \chi) / \partial(q, \Theta)| Q^{-1} \exp \{-q / Q\} \\
& =\left|\chi^{\prime}(\Theta)\right| Q^{-1} \exp (-q / Q),
\end{aligned}
$$

which is in fair agreement with plots depicted in Kurgansky and Tatarskaya (1987) and Pisnitchenko and

\footnotetext{
${ }^{2}$ Evidence of intersection between isentropic surfaces and the earth's surface brings neither conceptual nor technical problems, if using an artificial procedure proposed in (Lorenz 1955), that is, to complete the atmosphere by the weightless substance beneath the earth's surface. For such an extended system, "atmosphere + weightless gas underground", all the meteorological fields, including $\chi$ and $q$, can be continuously extrapolated below the earth's surface, and both the total atmospheric mass and vorticity charge conservation laws hold, automatically.
} 


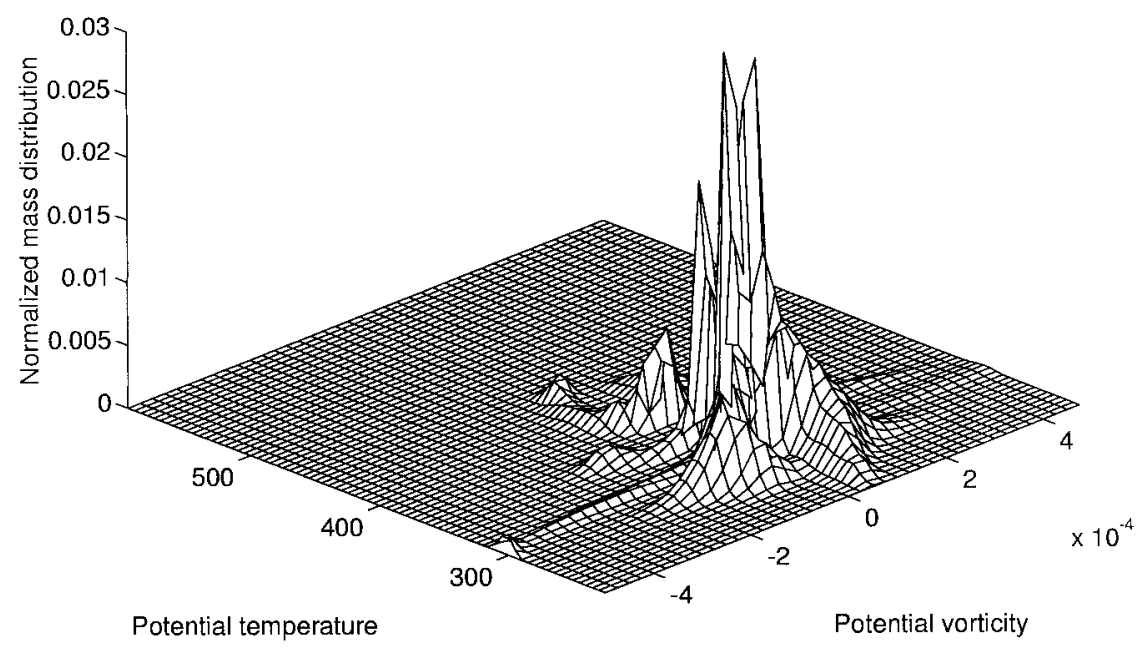

FIG. 2. Monthly mean atmospheric mass distribution on optimally modified PV and $\Theta$ for Jan 1980.

Kurgansky (1996), showing $\mu_{\mathrm{B}}(q, \Theta)$ maximum in vicinity of $\Theta=\Theta_{0}$ [see (3)], where $\left|\chi^{\prime}(\Theta)\right|=\max$ (Fig. 2).

It is worth mentioning that Kurgansky and Prikazchikov (1994) have demonstrated the closeness between actual monthly mean $\mu$ and reference $\mu_{\mathrm{B}}$ distribution for PV values computed after Kurgansky and Tatarskaya (1987) and Obukhov et al. (1988) with the use of climatological data for $\chi$ definition. Further on, $\mu(q, \chi)$ distribution from section $3 b$ will be reduced to onedimensional probability density $\mu(q)=\int \mu(q, \chi) d \chi$, by integrating over all $\chi$.

For both hemispheres separately, the introduced above distribution

$$
\mu_{\mathrm{B}}(q)=|Q|^{-1} \exp (-q / Q),
$$

where $q>0(Q>0)$ and $q<0(Q<0)$ corresponds to the case of the $\mathrm{NH}$ and the $\mathrm{SH}$, respectively, supplies the maximum of the informational entropy $H=-\int \mu$ $\ln \mu d q$, provided

$$
Q=\int q \mu_{\mathrm{B}}(q) d q=\int q \mu(q) d q=\text { constant. }
$$

We name $\mu_{\mathrm{B}}(q)$ the reference distribution. In the $H$ definition, a multiplicative constant is set equal to unity (without loss of generality), and an additive constant, for which a precise value is not significant, is omitted. The informational entropy $H$ maximum value is equal to

$$
H_{\mathrm{B}}=-\int \mu_{\mathrm{B}} \ln \mu_{\mathrm{B}} d q=\ln |Q|+\text { constant, }
$$

and the informational entropy deficit

$$
\Delta H=H_{\mathrm{B}}-H
$$

characterizes the degree of closeness between $\mu(q)$ and $\mu_{\mathrm{B}}(q)$ distributions. This is the measure of the amount of information that $\mu(q)$ distribution possesses in addition to the known $Q$ value. According to (7), $Q$ value completely determines $\mu_{\mathrm{B}}(q)$ distribution. So, when $\mu(q)$ coincides with $\mu_{\mathrm{B}}(q)$, then $\Delta H=H_{\mathrm{B}}-H_{\mathrm{B}} \equiv 0$ (see also Yaglom and Yaglom 1973).

One might hypothesize that the closer we approach to the optimal PV modification, the nearer $\mu(q)$ and $\mu_{\mathrm{B}}(q)$ distributions become and the smaller $\Delta H$ value is. Inversely, this gives a tool, how minimizing $\Delta H$ for different $\chi$ functions to arrive at the best choice of $\chi$, giving an optimally modified PV.

\section{d. Optimal potential vorticity modification}

In our search for the optimal function $p_{0}^{*}$ we allowed variations in $C$ and $\Theta_{0}$ in (3), but kept constant the parameter $B$ and $A=(\pi / 2) B$ values as $A=681 \mathrm{hPa}$, and $B=433.5 \mathrm{hPa}$. ECMWF 1980-89 data were used and for every calendar year both January and July monthly mean $\mu(q)$ distributions were calculated for 64 different pairs of $C$ and $\Theta_{0}$ values for the $\mathrm{NH}$ and $\mathrm{SH}$, separately. For numerical computations, Eqs. (A4)-(A6) with $\Delta q=$ $0.25 \times 10^{-4} \mathrm{~s}^{-1}, M=19$, and $k=(\ln 19)^{-1}$ were used. The values of $Q$, of informational entropy $H$, and of the difference $\Delta H=H_{\mathrm{B}}-H$ were also calculated and after that averaged over the entire 10 -yr period. As it is seen in Fig. 3, for both extreme months and for both hemispheres $\Delta H=\Delta H\left(\mathrm{C}, \Theta_{0}\right)$ attains minimum values in the vicinity of a line given by the linear regression equation $\Theta_{0}-292.55=321.4 \times(C-0.04614)$. Here, $\Theta_{0}$ is expressed in Kelvins $(\mathrm{K})$ and $C$ is given in $\mathrm{K}^{-1}$. Figure 4 shows that the position of the minimum for the corresponding standard deviations of $\Delta H$ coincides with that of the mean $\Delta H$ values, confirming that we have a statistically stable minimum. For brevity, only the plot for January NH is presented to illustrate this point. The parameters in (3) that supply the minimum of $\Delta H$ are plau- 
JANUARY NH, 1980-89 mean

(a)

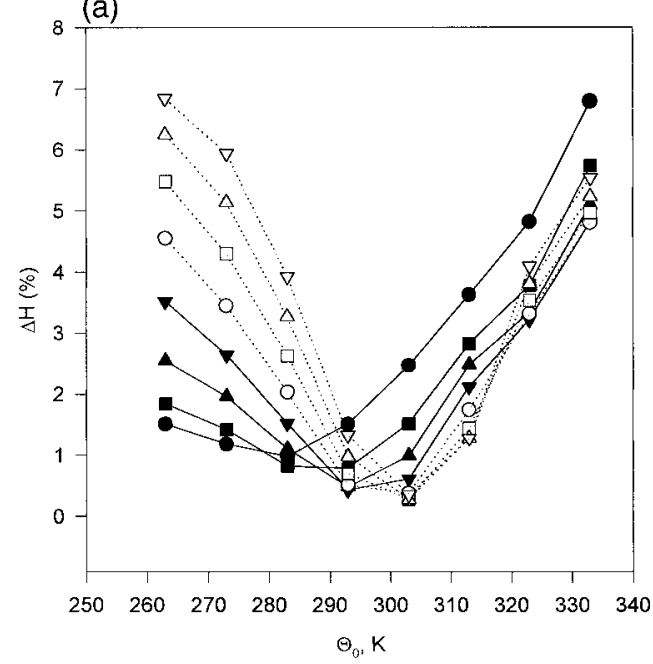

JULY NH, 1980-89 mean

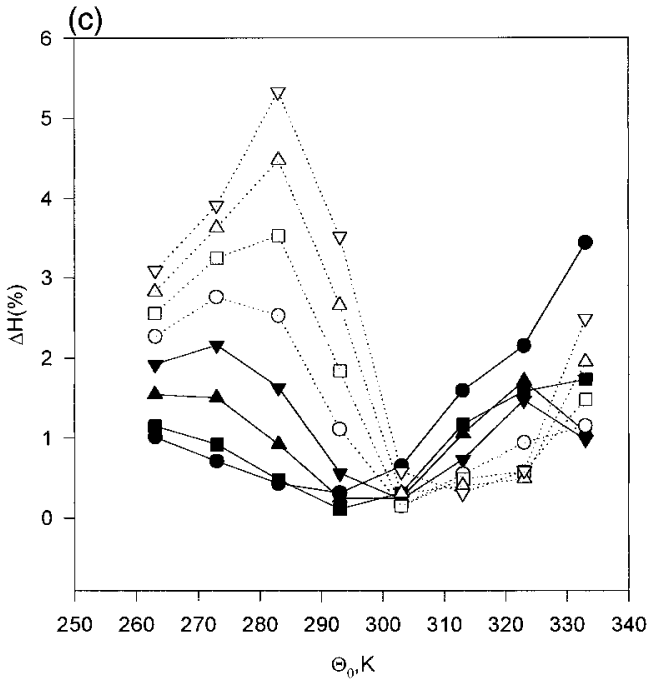

JANUARY SH, 1980-89 mean

(b)

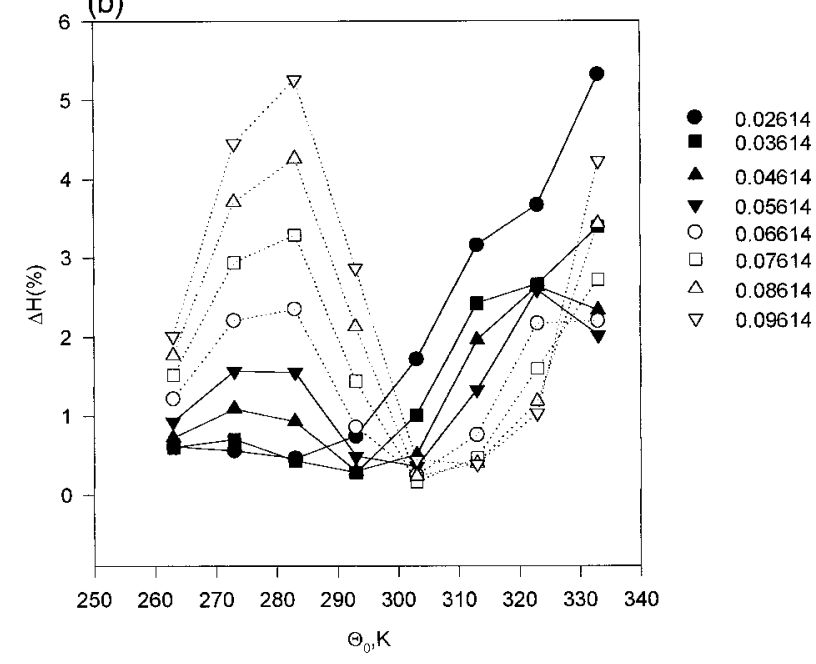

JULY SH, 1980-89 mean

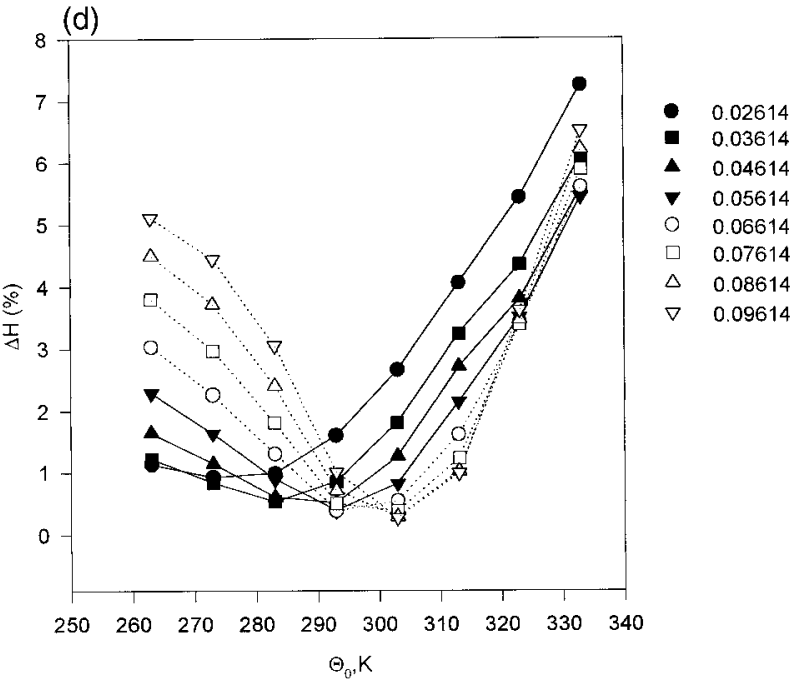

FIG. 3. (a) Dependence of informational entropy deficit $\Delta H$ on the potential temperature $\Theta=\Theta_{0}(\mathrm{~K})$, corresponding to the maximum of $\left|d p^{*}(\Theta) / d \Theta\right|$ in (3), for eight different values of the parameter $C\left(\mathrm{~K}^{-1}\right)$, which characterizes the temperature lapse rate. The case of $\mathrm{NH}$ for Jan is considered; $\Delta H$ are calculated using the monthly mean $\mu$ distribution for every year and then averaging them over 1980-89; computed $\Delta H$ values are multiplied by the factor of $10^{2}$ and expressed in percent (the latter show what the percentage of ln 19 the $\Delta H$ magnitude is). (b) The same as in (a) but for the SH, Jan; (c) the same as in (a) but for the NH, Jul; (d) the same as in (a) but for the SH, Jul.

sible candidates to be used for the optimal PV modification. In further analysis, the values $\Theta_{0}=293 \mathrm{~K}$ and $C=0.04614 \mathrm{~K}^{-1}$, lying close to the regression line, were chosen. Figure 5 demonstrates the evident closeness between actual $\mu(q)$ and reference $\mu_{\mathrm{B}}(q)$ distributions for such an optimal PV modification. Here, monthly mean $q$ statistics for January and July 1980-89 and for both the $\mathrm{NH}$ and $\mathrm{SH}$ were used. Systematic deviations occur only at large $q$ values. In the used semilogarithmic coordinates a straight line corresponding to $\mu_{\mathrm{B}}(q)$ nearly coincides with a straight line that best fits the $\mu(q)$ curve in the least squares sense. The closeness between $\mu$ and $\mu_{\mathrm{B}}$ lines and especially their divergence at large $q$ values may serve as a hint for construction of the forthcoming grounds for the $\mu_{\mathrm{B}}(q)$ distribution justification.

When we calculate the $\mu$ function for a hemispheric atmosphere, then in quite a random manner we choose an air parcel and after that evaluate what MPV value it has. This procedure is equivalent to the consideration of an ensemble of hypothetical atmospheres over the hemisphere. Every representative (elementary state) of this ensemble is supposed to have a spatially uniform MPV field, characterized by a single $q$ value. If the total hemispheric mass is set equal to unity (which is always possible by mass rescaling), then the hemispheric vorticity charge is equal to $q$ also. The mathematical ex- 
JANUARY NH, 1980-89 standard deviation

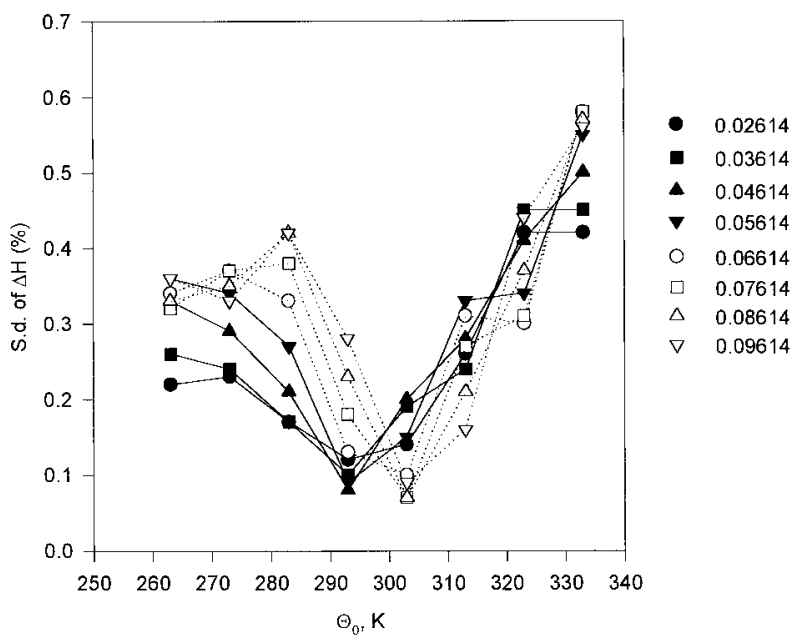

FIG. 4. Standard deviations (s.d.) of $\Delta H$ from their mean values for 1980-89. Other notations are as in Fig. 3; NH, Jan.

pectation value of vorticity charge $q$ for such the ensemble is a prescribed constant quantity, which is assumed be equal to $Q$. [This is the exact analog of how a canonical (in Gibbs's sense) statistical distribution for a system in a thermostat is introduced.] Now, the probability density $\mu$ of MPV values for a randomly chosen air parcel coincides exactly with the probability density of vorticity charge values $q$ for the ensemble of elementary states. Any elementary state, having the vorticity charge $q$, can be decomposed into a sum of two independent elementary states with vorticity charges $q_{1}$ and $q_{2}$, such that $q=q_{1}+q_{2}$. Now, from the probability calculus the functional equation $\mu\left(q_{1}+q_{2}\right)=$ $\mu\left(q_{1}\right) \mu\left(q_{2}\right)$ follows. After solving it, we arrive at the needed exponentially decaying $\mu_{\mathrm{B}}$ distribution, which serves as a canonical one for the ensemble in question. ${ }^{3}$ Canonical distribution has the highest informational entropy $H$ value if compared to all other virtual $q$ distri-

\footnotetext{
${ }^{3}$ Due to the lower atmosphere radiative heating, and a subsequent diabatic destruction of PV field, there is a permanent tendency toward atmospheric mass concentration at zero PV values [cf. Danielsen (1990), where, inversely, PV production in the stratosphere due to diabatic cooling is discussed extensively]. The corresponding limit state, with entire air mass over a hemisphere being concentrated at zero PV value, would realize for vanishing meridional diabatic heating gradient, when in the atmospheric bulk one observes only microscale and mesoscale motions with identically vanishing PV field, which originate from vertical convective instability. In this hypothetical state, the hemispheric vorticity charge $q=0$. Work of atmospheric general circulation, driven by differential diabatic heating, results in the production of $q>0$ (for the $\mathrm{NH}$ ). In the frame of this scheme, it is natural to assume that in order to create vorticity charge $q_{1}+q_{2}>0$ it is necessary to create, at first step, the vorticity charge $q_{1}>0$ and after that the vorticity charge $q_{2}>0$. If these two steps are independent, one arrives at the functional relation between probability densities from the main body of the text.
}

butions between elementary states from this ensemble. The strict justification of the possibility of transferring the results obtained for this ensemble into the case of atmospheric MPV statistics demands on the ergodicity assumption or of very strong mixing occurring in the MPV functional space [sometimes referred to as K-mixing, named after Kolmogorov (Dymnikov and Filatov 1997)]. In practical terms, it might mean that if we choose any fixed geographic position in the atmosphere, then the corresponding local MPV value should pass (during a sufficiently long but still finite time interval) through nearly all MPV from the entire range of their possible values. Notice here that tropospheric MPV values may be as high as $8 \times 10^{-4} \mathrm{~s}^{-1}$ in cyclones, and as low as (0.3-0.5) $\times 10^{-4} \mathrm{~s}^{-1}$ in anticyclones (Kurgansky and Tatarskaya 1987), and, what is more, these synoptic vortices can travel freely over any arbitrary but fixed geographic point in middle and high latitudes. In other words, the observed variability in MPV terms is greater than that in terms of any other known meteorological variable, and just this property ensures the success in applying statistical methods to MPV field. As an example, we give here a plot of MPV temporal behavior in two different but closely spaced geographic positions. One might easily see in Fig. 6 that MPV temporal variations are quasi-random and are not cross-correlated in the taken points. Because of the use of essentially massweighted statistics, a role of the stratosphere is masked and the stress is made on the troposphere where the main temporal and spatial variability in the MVP field occurs. In the real atmosphere, the MPV field is spatially nonuniform and there is no global statistical equilibrium for this variable, in this particular sense. Nevertheless, in any fixed geographic point we may have a local statistical equilibrium.

In the hypothetical state of full statistical equilibrium over a hemisphere, due to ergodicity, in every fixed geographic position one would have distribution (7) for the frequency of $q$ values' occurrence with a time-mean value of $q$ equal to $Q$. Under the assumption of an infinitesimally small radius of spatial correlation in $q$ field and envoking ergodicity theorems once more, it results at distribution (7) for the entire hemispheric atmosphere. It is more difficult to prove the reverse assertion. Nevertheless, a close inspection of Fig. 6 gives the strong hint that in nearly every geographic position within the extratropical troposphere, the $q$ histogram will closely follow (7) if only to replace $Q$ with a corresponding local time-mean $q$ value.

The functional relation $\mu\left(q_{1}+q_{2}\right)=\mu\left(q_{1}\right) \mu\left(q_{2}\right)$ stands for the probability of a realization of two independent events simultaneously. A question arises of whether or not we can always (i.e., for any $q$ ) consider an elementary $q$ state as a superposition of two independent states. Inversely, if we have two elementary states with large $q_{1}$ and $q_{2}$ values (two states with strong cyclonic circulation), can we judge that the state with $q=q_{1}+q_{2}$ will be an achievable atmospheric state? 

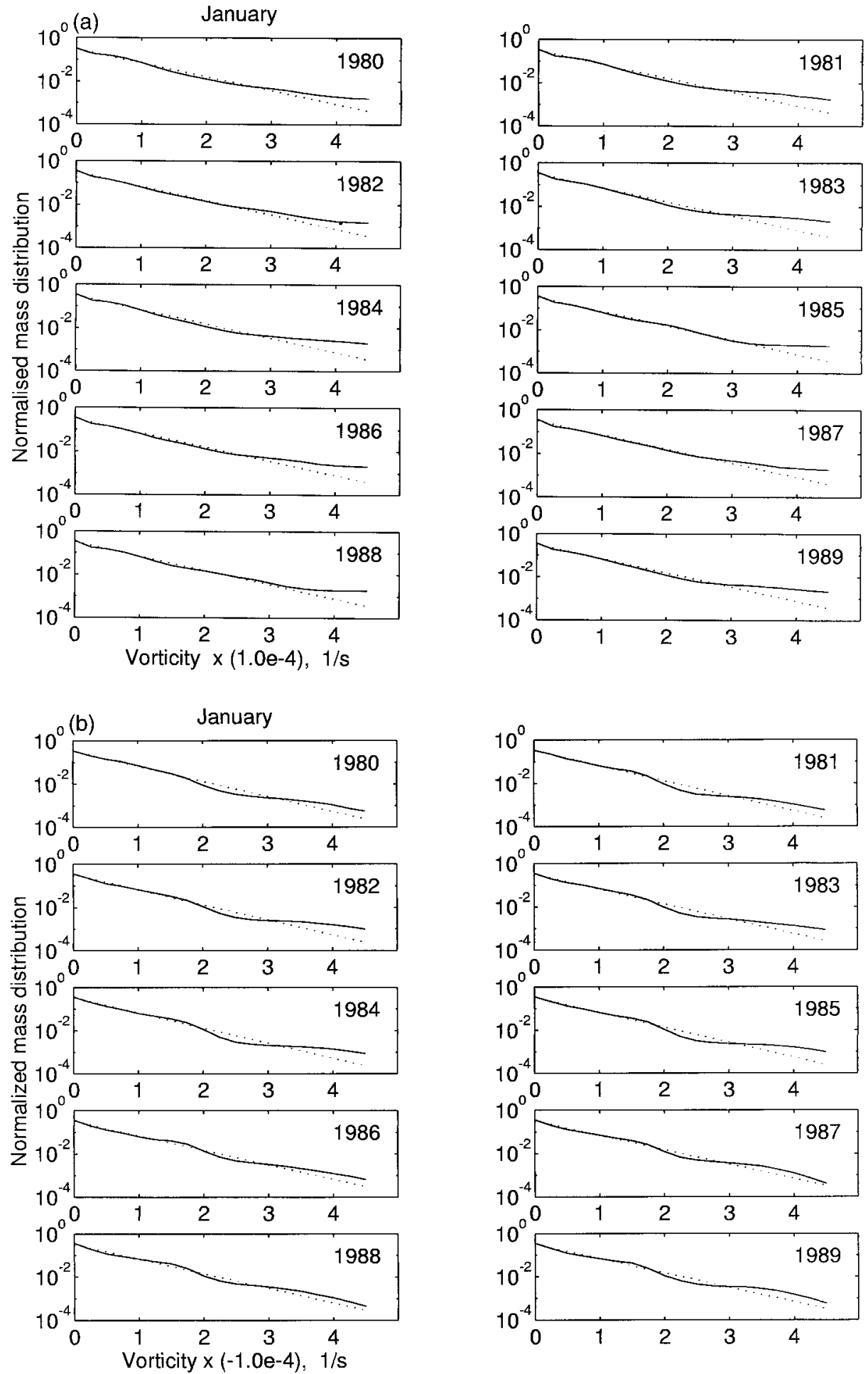

FIG. 5. Atmospheric mass distribution on optimally modified potential vorticity for Jan 1980-89 (a) NH and (b) SH; and Jul 1980-89. 

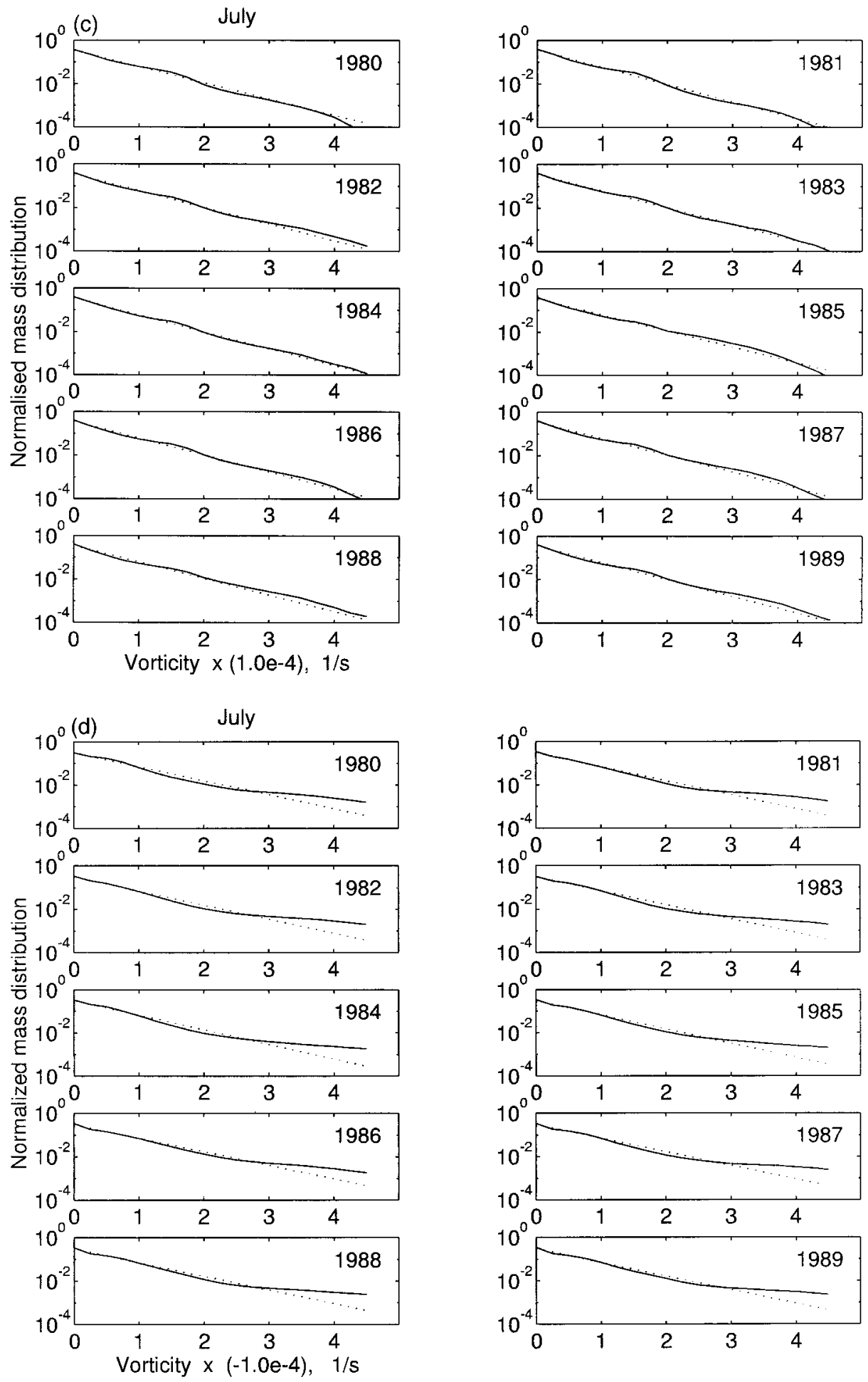

FIG. 5. (Continued) (c) NH and (d) SH. Solid line corresponds to the actual distribution, dotted line to the reference distribution. 

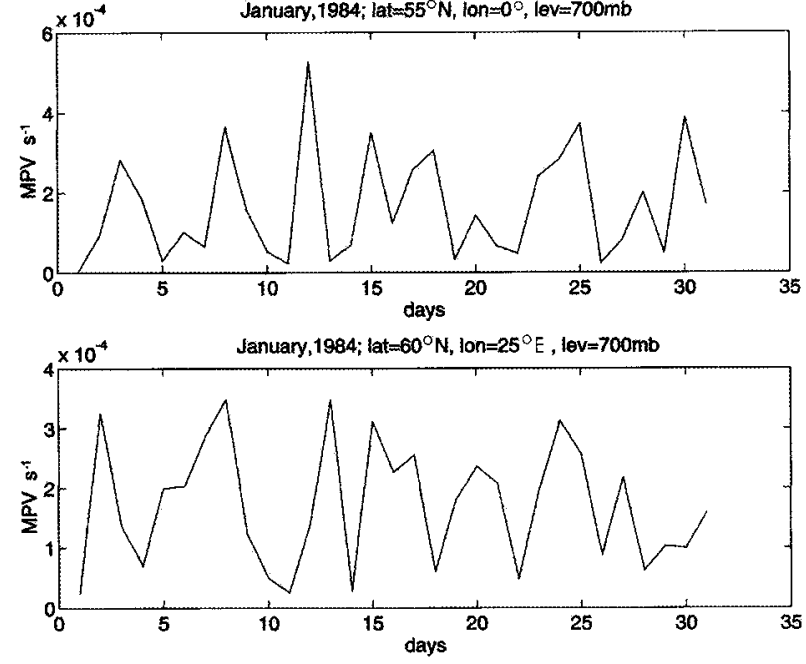

FIG. 6. Temporal evolution of modified potential vorticity $q$ in two fixed geographical points with coordinates (lat $=55^{\circ} \mathrm{N}$, lon $=0^{\circ}$, lev $=700 \mathrm{mb}$; and lat $=60^{\circ} \mathrm{N}$, lon $=25^{\circ} \mathrm{E}$, lev $=700 \mathrm{mb}$ ) during Jan 1984.

One can affirm that, because of the existing upper limit for MPV, there are physical restrictions for the sum $q_{1}$ $+q_{2}$ to represent a real atmosphere. Therefore, one might expect a priori certain discrepancies between $\mu$ and $\mu_{\mathrm{B}}$ distributions for large $q$ values. Figure 5 distinctly demonstrates this circumstance, showing that $\mu$ values slightly exceed $\mu_{\mathrm{B}}$ ones in the $q$ range in question.

We want to notice that the resulting reference pressure $p_{0}^{*}$ profile is in fair agreement with that previously used by Dutton and Johnson (1967) in Lorenz's (1955) theory of available potential energy, and by Tatarskaya (1978), Kurgansky and Tatarskaya (1987), and Obukhov et al. (1988) in earlier potential vorticity computations. Here, we remark only that the formula $\gamma_{a}-\gamma\left(\Theta_{0}\right)=$ (7 $\pi / 4) \gamma_{a} \Theta_{0}^{-1} C^{-1}$ (Kurgansky and Tatarskaya 1987), where $\gamma_{\mathrm{a}}$ is the dry-adiabatic lapse rate and $\gamma$ is the temperature lapse rate, which corresponds to the $p_{0}^{*}$ profile, is readily derived from (3) at $\Theta=\Theta_{0}$. For the chosen $C$ and $\Theta_{0}$ values, it gives $\gamma \approx 5.9 \times 10^{-3} \mathrm{~K}$ $\mathrm{m}^{-1}$, which is in accordance with observations.

Temporal behavior of the informational entropy $H$ for $\Theta_{0}=293 \mathrm{~K}$ and $C=0.04614 \mathrm{~K}^{-1}$ within the 1980 89 period is presented in Fig. 7. Corresponding numerical values are given in Table 1 together with $H_{\mathrm{B}}$, $\Delta H=H_{\mathrm{B}}-H$, and $Q$ estimates. We can see that the entropy deficit $\Delta H$ evolves within an interannual time range, keeping stable its order of magnitude.

\section{Concluding remarks}

We have shown that by using the existing arbitrariness in a general Ertel's PV definition it is possible to construct a modified potential vorticity $q$, which corresponds to the highest possible degree of closeness between actual and reference atmospheric airmass distributions of potential

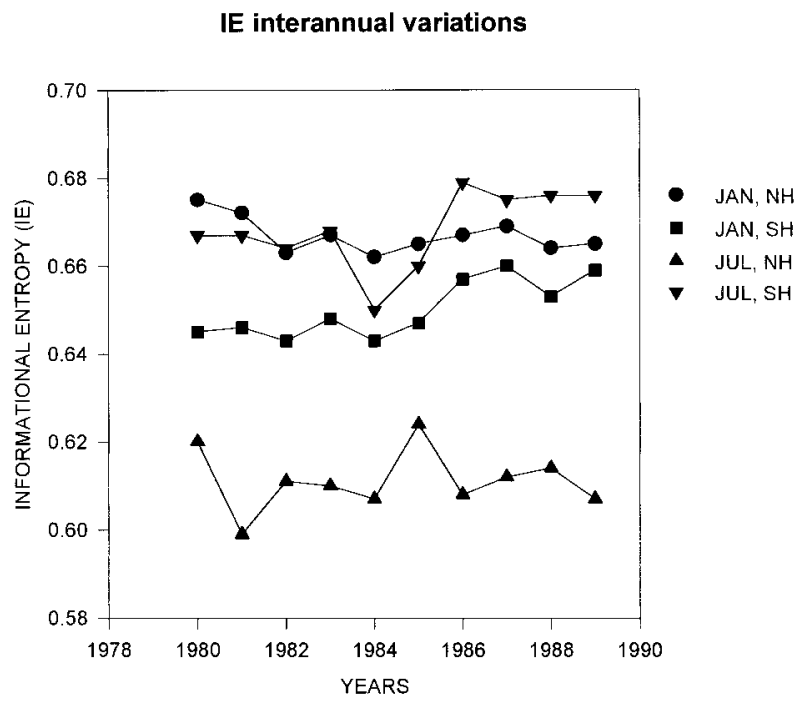

FIG. 7. Interannual variability of the informational entropy $H$ of monthly mean airmass distribution on optimally modified potential vorticity for $1980-89$.

vorticity. The latter has the same amount (per unit mass) of the vorticity charge $Q$ over a hemisphere as actual atmospheric state does and gains the maximum value of the informational entropy. The optimal choice of an arbitrary function of potential temperature $\chi(\Theta)=$ $-p_{0}^{*}(\Theta) / g$, entering the $q$ definition, results in a twodimensional $\mu(q, \chi)$ distribution, which is fairly close to an idealized statistically equilibrium distribution $\mu_{\mathrm{B}}(q)$, having no dependence on $\chi$, that is, with equipartitioning of atmospheric mass between equal intervals of $\chi$. By this reasoning, we have reduced the two-dimensional $\mu(q, \chi)$ distribution to a one-dimensional distribution $\mu(q)=\int \mu(q, \chi) d \chi$, used in this paper.

The fact of independence of a reference distribution (7) upon the coordinate $\chi$ even looks trivial a posteriori. If $p_{0}^{*}(\Theta)$ happens to be close to the average hydrostatic pressure on isentropic surfaces, then there should be near equipartitioning of air mass between atmospheric layers separated with unit isoscalar surfaces $\chi_{0}, \chi_{0}+$ $1, \chi_{0}+2, \ldots$ Certain deviations, possibly visible in Fig. $1 \mathrm{~b}$, are caused by imperfections in choice of a "trial function" [Eq. (3)]. To construct a more "perfect" approximation for $\chi(\Theta)$, which nevertheless admits a simple enough analytical representation to be effectively used in informational entropy deficit minimum searching, remains a future task.

Our numerical calculations give a hint that a hypothetical hydrostatic atmospheric state, which is completely determined by the $\chi(\Theta)$ function, is fairly close to the reference state appearing in the Lorenz's (1955) available potential energy (APE) theory. To answer the question of whether this coincidence is accidental or whether there are some fundamental physical reasons for it remains a task for the future. Following the pro- 
TABLE 1. Interannual variations of the intergrals of potential vorticity field.

\begin{tabular}{|c|c|c|c|c|c|c|c|c|c|}
\hline Year & $H$ & $H_{\mathrm{B}}$ & $10^{2} \times\left(H_{\mathrm{B}}-H\right)$ & $Q \times 10^{4} \mathrm{~s}^{-1}$ & Year & $H$ & $H_{\mathrm{B}}$ & $10^{2} \times\left(H_{\mathrm{B}}-H\right)$ & $Q \times 10^{4} \mathrm{~s}^{-1}$ \\
\hline \multicolumn{5}{|c|}{ NH, Jan } & \multicolumn{5}{|c|}{ NH, Jul } \\
\hline 1980 & 0.675 & 0.678 & 0.360 & 0.682 & 1980 & 0.620 & 0.622 & 0.166 & 0.579 \\
\hline 1981 & 0.672 & 0.678 & 0.535 & 0.680 & 1981 & 0.599 & 0.601 & 0.219 & 0.545 \\
\hline 1982 & 0.663 & 0.667 & 0.356 & 0.659 & 1982 & 0.611 & 0.613 & 0.213 & 0.565 \\
\hline 1983 & 0.667 & 0.673 & 0.534 & 0.671 & 1983 & 0.610 & 0.612 & 0.182 & 0.563 \\
\hline 1984 & 0.662 & 0.668 & 0.539 & 0.661 & 1984 & 0.607 & 0.609 & 0.125 & 0.557 \\
\hline 1985 & 0.665 & 0.669 & 0.372 & 0.663 & 1985 & 0.624 & 0.626 & 0.240 & 0.586 \\
\hline 1986 & 0.667 & 0.673 & 0.541 & 0.670 & 1986 & 0.608 & 0.611 & 0.265 & 0.561 \\
\hline 1987 & 0.669 & 0.675 & 0.535 & 0.674 & 1987 & 0.612 & 0.616 & 0.359 & 0.568 \\
\hline 1988 & 0.664 & 0.670 & 0.472 & 0.664 & 1988 & 0.614 & 0.619 & 0.415 & 0.573 \\
\hline 1989 & 0.665 & 0.671 & 0.594 & 0.668 & 1989 & 0.607 & 0.611 & 0.321 & 0.560 \\
\hline \multicolumn{5}{|c|}{ SH, Jan } & \multicolumn{5}{|c|}{ SH, Jul } \\
\hline 1980 & 0.645 & 0.648 & 0.194 & -0.624 & 1980 & 0.667 & 0.673 & 0.527 & -0.671 \\
\hline 1981 & 0.646 & 0.648 & 0.200 & -0.624 & 1981 & 0.667 & 0.671 & 0.412 & -0.667 \\
\hline 1982 & 0.643 & 0.646 & 0.312 & -0.621 & 1982 & 0.664 & 0.670 & 0.500 & -0.665 \\
\hline 1983 & 0.648 & 0.651 & 0.288 & -0.630 & 1983 & 0.668 & 0.673 & 0.479 & -0.671 \\
\hline 1984 & 0.643 & 0.647 & 0.283 & -0.621 & 1984 & 0.650 & 0.655 & 0.489 & -0.638 \\
\hline 1985 & 0.647 & 0.650 & 0.308 & -0.628 & 1985 & 0.660 & 0.666 & 0.496 & -0.657 \\
\hline 1986 & 0.657 & 0.661 & 0.314 & -0.647 & 1986 & 0.679 & 0.684 & 0.457 & -0.694 \\
\hline 1987 & 0.660 & 0.663 & 0.287 & -0.652 & 1987 & 0.675 & 0.682 & 0.637 & -0.688 \\
\hline 1988 & 0.653 & 0.658 & 0.454 & -0.643 & 1988 & 0.676 & 0.682 & 0.550 & -0.689 \\
\hline 1989 & 0.659 & 0.664 & 0.396 & -0.653 & 1989 & 0.676 & 0.681 & 0.543 & -0.688 \\
\hline
\end{tabular}

posed procedure, further expanded computational experiments are needed, probably using a broader class of $p^{*}(\Theta)$ functions than those represented by (3).

We want to stress that the idea underlying our work is "kinematically" very similar to that which is put into the basis of APE concept (Lorenz 1955). In the frame of APE theory, the hypothetical atmospheric reference state, which is mechanically absolutely stable, contains a major part of total potential energy (TPE) of the atmosphere, but this amount of energy, called the unavailable potential energy, cannot be be converted into kinetic energy. Only a minor residual part of TPE, named APE, is able to go on doing mechanical work. The ratio APE/TPE happens be very small and it could serve as a small nondimensional parameter that measures the degree of global nonequilibrium of the atmosphere in the terrestrial gravity field and its ability to produce mechanical work (the atmosphere to work as a heat engine). In the problem considered here, we have an analogously small parameter, which describes small deviations of the atmosphere from the state of complete statistical equilibrium if expressed in terms of functionals of modified potential vorticity $q$. This small parameter is specified by the ratio of informational entropy deficit to informational entropy itself. The latter quantity is a cumulative and concise characterization of atmospheric mass distribution on MPV values. In every hemisphere it reaches maximum value for a hypothetical reference stationary statistical regime, which is completely defined by a sole physical parameter over a hemisphere, namely, the atmospheric vorticity charge $Q$. Likewise, nonvanishing of APE is caused by temperature (pressure) nonuniformity along isentropic surfaces; the nonzero informational entropy deficit is explained by time-mean MPV field nonhomogeneity in horizontal (predominantly meridional) direction. The latter conclusion is routed in the used PV modification, because this quantity mainly varies in the equator-to-pole direction.

It does not seem to be a deficiency of our approach that both $\mu(q)$ and $\mu_{\mathrm{B}}(q)$ distributions are defined and analyzed for the hemispheres separately but not globally. First, following the modern point of view, the climate changes having either natural or man-made origin could be detected using "fingerprint" asymmetries between the hemispheres. Second, when considering the hemispheric monthly mean $\mu(q)$ statistics, we have all the reasons to neglect the cross-equatorial interhemispheric airmass exchange, which is significant, starting only from the annual or more time period, as the recent data on global diffusion of various air pollutants clearly show.

Finally, a question might arise, how does the state of complete statistical equilibrium, described by the reference distribution $\mu_{\mathrm{B}}(q)$, "look" in commonly used geographical coordinates? If $q$ values in different positions are not cross-correlated, that is, $q$ field has infinitesimally small spatial radius of correlation, then for any atmospheric subdomain (parcel) we will have a probability distribution $\mu_{\mathrm{B}}(q)$, with a mathematical expectation value of $q$ equal to $Q$. Necessarily, for the entire hemispheric atmosphere the average MPV value equals $Q$ as well, and integral constraint of vorticity charge conservation is not violated. In this particular, statistically averaged sense, one might assert that in geometric coordinates the reference distribution $\mu_{\mathrm{B}}(q)$ corresponds to Rossby's (1947) fully horizontally homogenized (mixed) hemispheric atmosphere state. Naturally, horizontal MPV fluxes vanish identically in this regime of complete statistical 
equilibrium ${ }^{4}$. Actual atmospheric state, with horizontal gradients in time-mean MPV field, is characterized by local statistical equilibrium and is observed in close vicinity of complete (global) statistical equilibrium over a hemisphere. Physically, the corresponding measure of deviation from the reference state is characterized by the intensity of time-mean MPV fluxes. The main finding of this paper is that for a reasonably, that is, optimally, modified PV concept the degree of horizontal inhomogeneity in the MPV field happens to be fairly small (if measured in units of informational entropy deficit), and we can effectively treat the atmospheric climate system as nearly statistical equilibrium. In perspective, it could serve as a tool for additional justification for a commonly used "downgradient" parameterization of time-mean potential vorticity eddy fluxes (cf. Kurgansky and Tatarskaya 1987 and references therein). It should be noted, in conclusion, that the request of smallness of informational entropy deficit $\Delta H$ is much less restrictive than the demand for smallness of the ratio $\Delta q / Q$. Here, $\Delta q$ is the equator-to-pole difference in time-mean $q$ values. Actually, $\Delta q / Q=O(1)$, but nevertheless, $\Delta H$ happens to be small enough if compared to unity, as we have seen before. This is the only circumstance that makes the aboveused analogy with Lorenz's APE theory not straightforward to the very end, because the smallness of APE implies the smallness of the corresponding ratio for temperature field as well.

The optimally modified PV happens be the closest in the least squares sense to the quasigeostrophic potential vorticity (QGPV) in isentropic coordinates (Kurgansky 1973; Berrisford et al. 1993), though the MPV field does not coincide identically with that of QGPV, especially in low latitudes and in extreme points of the PV field. Our computations reveal that extreme MPV values are

\footnotetext{
${ }^{4}$ When following directly Rossby's (1947) scheme, one should assume the MPV field to be completely homogenized (mixed) only poleward to certain critical lalitudes, one lying in $\mathrm{NH}$ and the other in SH. Between these latitudinal circles an equatorial transitional zone has to be, with cross-equatorial MPV fluxes directed from $\mathrm{NH}$ to $\mathrm{SH}$. In this context, one can say that it is difficult to construct a regime of complete statistical equilibrium for the entire terrestrial atmosphere. Nevertheless, for the hemispheres considered separately it could be done successfully. Allowing once more an electrostatic analogy (Bishop and Thorpe 1994; Thorpe and Bishop 1995), one might figuratively say that the separation of hemispheres is analogous to insulation from each other of two electrostatic charges of opposite polarities, when the electric current between them leading to this "global condenser" discharge is forbidden.

In the vicinity of the equator, in the main tropospheric bulk, $q$ values are increasingly small. Moreover, one regularly observes air parcels with negative $q$ at low latitudes in the $\mathrm{NH}$ and similarily those with positive $q$ in the SH. So in an anually averaged sense one might say that an equatorial zone with nearly uniform vanishing $q$ values and hence zero MPV meridional fluxes creates a "locking layer," which blocks up the interhemispheric MPV exchange and helps to consider the hemispheric atmospheres separately. Recalling again the electrostatic analogy, one might say that this is equivalent to the positioning of a thin insulator film between two neighboring electric charges of opposite sign.
}

predominantly caused by static stability anomalies. From the other side, for synoptic-scale motions the contribution of relative vorticity and static stability variations to the QGPV value is approximately the same, or of the same order of magnitude, at least. As a result, one might expect noticeable discrepancies between our statistical results and those that would be obtained for the QGPV field. To compare atmospheric mass statistics for MPV and QGPV, respectively, remains an interesting problem for future investigations.

Acknowledgments. The authors owe an incredible debt to the late Professor A. M. Obukhov, whose many ideas lie in the heart of this investigation. We are obliged to Prof. V. P. Dymnikov and Drs. I. I. Mokhov and V. K. Petoukhov for helpful discussions, and to Dr. M. Tippett for reading the manuscript and making valuable remarks. The financial support of this research by the Brazilian National Council for Development of Science and Technology is gratefully acknowledged. We are grateful for the support from the Russian Foundation for Basic Research under project Grants 96-15-98527 and 96-05-64511.

\section{APPENDIX}

\section{Discrete Version of the Reference Distribution}

In practice, the distribution $\mu(q)$ is known in the form of a table $\mu_{i}, i=1, \ldots, M$, such that

$$
\sum_{i=1}^{M} \mu_{i}=1
$$

Each cell corresponds to an interval $\Delta q$ on the $q$ axis starting from zero. Hereafter, only positive $q$ are considered. This is the case of the Northern Hemisphere, an extension onto the case of the Southern Hemisphere being evident. Informational entropy is given by

$$
H=-k \sum_{i=1}^{M} \mu_{i} \ln \mu_{i} .
$$

Commonly, it is adopted that $M=$ constant, and $k=$ $(\ln M)^{-1}$. Informational entropy, thus normalized, attains its maximum value $H_{\max }=1$ for the case of air mass equipartitioning between the cells.

Let us assume that the number of cells $M$ is not fixed but arbitrary, instead. Only the assumption $M \gg 1$ is made. One seeks the maximum of (A2) when conditions (A1) and

$$
\sum_{i=1}^{M} q_{i} \mu_{i}=Q=\text { constant }
$$

are satisfied. Here, $q_{i}$ is the potential vorticity value in the middle of the $i$ th cell, the differences between $q$ values within the cell being neglected. It means that $\Delta q$ is small enough. From the other side, one should keep in mind a general limitation of classic statistics: a number $n_{i}$ of air parcels with $q$ values falling into the $i$ th 
cell must satisfy the necessary condition $n_{i} \gg 1$. As a result, $\Delta q$ cannot be taken too small, and a compromise choice of $\Delta q$ must be made in the course of practical computations.

Boltzmann (1978) solved a very similar variational problem as early as in 1877 . His solution results in a geometric progression $\mu_{i}^{\mathrm{B}}=\mu_{1}^{\mathrm{B}} \alpha^{i-1}$, with the denominator $\alpha$ being determined in our case through the equation

$Q=\frac{\Delta q}{2}+\Delta q \alpha \frac{1-\alpha}{1-\alpha^{M}} \cdot \frac{1-M \alpha^{M-1}+(M-1) \alpha^{M}}{(1-\alpha)^{2}}$

It also holds that $\mu_{1}^{\mathrm{B}}=(1-\alpha)\left(1-\alpha^{M}\right)^{-1}$. For $M \gg$ 1, when both $\alpha^{M} \ll 1$ and $M \alpha^{M} \ll 1$, it follows that

$$
\mu_{1}^{\mathrm{B}} \approx 1-\alpha, \quad Q \approx(\Delta q / 2)(1+\alpha)(1-\alpha)^{-1},
$$

and as a result

$$
\alpha=(2 Q-\Delta q) /(2 Q+\Delta q) ;
$$

that is, the reference distribution $\mu_{1}^{\mathrm{B}}$ is completely determined by $Q$ and $\Delta q$ values (actually by their ratio $Q$ / $\Delta q$ ). Informational entropy in the reference state is equal to

$$
H_{\mathrm{B}}=-k\left[\ln \frac{1-\alpha}{1-\alpha^{M}}+\left(\frac{Q}{\Delta q}-\frac{1}{2}\right) \ln \alpha\right],
$$

where $Q$ and $\alpha$ are linked via (A3). For the case of $M$ $\gg 1$ one has

$$
H_{\mathrm{B}} \approx-\frac{k}{1-\alpha}[(1-\alpha) \ln (1-\alpha)+\alpha \ln \alpha] .
$$

Further on, we restrict ourselves within this limiting case. It is useful to introduce a new variable, $\beta=\alpha(1$ $-\alpha)^{-1}$, such that $\beta=(\mathrm{Q} / \Delta q)-0.5, H_{\mathrm{B}}=k \ln [(1+$ $\left.\beta)^{1+\beta} \beta^{-\beta}\right]$, and hence $d H_{\mathrm{B}} / d \beta=k \ln [(1+\beta) / \beta]$. We define an auxiliary variable $\vartheta$ using a formal relation $\vartheta^{-1}=d H_{\mathrm{B}} / d Q$. Some straightforward algebraic transformations result in

$$
Q=(\Delta q / 2)+\Delta q\left[\exp \left(k^{-1} \vartheta^{-1} \Delta q\right)-1\right]^{-1} .
$$

When $\Delta q \ll 2 k \vartheta$, then $Q \approx k \vartheta$ and $H_{\mathrm{B}} \approx k \ln Q+$ const. The latter relation coincides with (9) in the special case of $k=1$.

\section{REFERENCES}

Berrisford, P., J. C. Marshall, and A. A. White, 1993: Quasigeostrophic potential vorticity in isentropic coordinates. J. Atmos. Sci., 50, 778-782.

Bishop, C. H., and A. J. Thorpe, 1994: Potential vorticity and the electrostatic analogy: Quasi-geostrophic theory. Quart. J. Roy. Meteor. Soc., 120, 713-731.

Boltzmann, L., 1978: Ueber die Beziehung zwischen den zweiten Hauptsatze der Mechanischen Waermetheorie und der Wahrscheinlichkeitsrechnung, respective den Saetzen ueber das Waermegleichgewicht (On the relation between the second principle of the mechanical theory of heat and the probability theory, respective to the theorems on heat equilibrim). Sitzungsber Akad. Wiss. Wien, 2 (76), 373-435.
Danielsen, E. F., 1990: In defense of Ertel's potential vorticity and its general applicability as a meteorological tracer. J. Atmos. Sci., 47, 2013-2020.

Dutton, J. A., and D. R. Johnson, 1967: The theory of available potential energy and a variational approach to atmospheric energetics. Advances in Geophysics, Vol. 12, Academic Press, 333.

Dymnikov, V. P., and A. N. Filatov, 1997: Mathematics of Climate Modeling. Birkhaeuser, $264 \mathrm{pp}$.

Ertel, H., 1942: Ein neuer hydrodynamischer Wirbelsatz. Meteor. Z., 59, 277-281.

Friedmann, A. A., 1934: Opyt Gidtomekhaniki Szhimaemoy Zhidkosti (Essay on the hydrodynamics of a compressible fluid). ONTI-GTTI, 370 pp.

Gibbs, J. W., 1948: Elementary principles in statistical mechanics, developed with especial reference to the rational foundation of thermodynamics. The Collected Works of J. Willard Gibbs, Vol. 2, Yale University Press, $207 \mathrm{pp}$.

Haynes, P. H., and M. E. McIntyre, 1990: On the conservation and impermeability theorems for potential vorticity. J. Atmos. Sci., 47, 2021-2031.

Hollmann, G. H., 1964: Ein vollstaendiges System hydrodynamisher Erchaltungssaetze. Archiv Meteor. Geophys. Bioklimatol., 14A, $1-13$.

Holton, J. R., P. H. Haynes, M. E. McIntyre, A. R. Douglass, R. B. Rood, and L. Pfister, 1995: Stratosphere-troposphere exchange. Rev. Geophys., 33, 403-439.

Hoskins, B. J., M. E. McIntyre, and A. W. Robertson, 1985: On the use and significance of isentropic potential vorticity maps. Quart. J. Roy. Meteor. Soc., 111, 877-946.

Katz, A., 1967: Principles of Statistical Mechanics. W. N. Freeman, $240 \mathrm{pp}$.

Koshyk, J. N., and N. A. McFarlane, 1996: The potential vorticity budget of an atmospheric general circulation model. J. Atmos. Sci., 53, 550-563.

Kurgansky, M. V., 1973: Calculation of the adiabatically equivalent zonal circulation of the atmosphere. Izv. Atmos. Ocean. Phys., 9, 875-877.

, 1991: The atmospheric vorticity charge. Izv. Atmos. Ocean. Phys., 27, 510-516.

— , and M. S. Tatarskaya, 1987: The potential vorticity concept in meteorology: A review. Izv. Atmos. Ocean. Phys., 23, 587-606.

— spheric seasonal variability indicator. Izv. Atmos. Ocean. Phys., 30, 730-737.

Lait, L. R., 1994: An alternative form for potential vorticity. J. Atmos. Sci., 51, 1754-1759.

Lorenz, E. N., 1955: Available potential energy and the maintenance of the general circulation. Tellus, 7, 157-167.

- 1995: The Essence of Chaos. University of Washington Press, $227 \mathrm{pp}$.

Nakamura, N., 1995: Modified Lagrangian-mean diagnostics of the stratospheric polar vortices. Part I: Formulation and analysis of GFDL SKYHI GCM. J. Atmos. Sci., 52, 2096-2108.

Obukhov, A. M., 1962: The dynamics of a stratified liquid. Sov. Phys. Dokl., 7, 682-684.

- 1964: Adiabatic invariants of atmospheric processes. Meteor. Gidrol., 2, 3-9.

— M. V. Kurgansky, and M. S. Tatarskaya, 1988: Isentropic analysis of global atmospheric processes using the field of potential vorticity from FGGE data. Meteor. Gidrol., 8, 111-120.

Pisnitchenko, I., and M. Kurgansky, 1996: Adiabatic invariants and diagnostic studies of climate. Ann. Acad. Bras. Cienc., 68 (1), 261-277.

Rossby, C. G., 1947: On the distribution of angular velocity in gaseous envelopes under the influence of large-scale horizontal mixing processes. Bull. Amer. Meteor. Soc., 28, 53-58.

Tatarskaya, M. S., 1978: On the adiabatic invariant distribution in the atmosphere of the Northern Hemisphere. Izv. Atmos. Ocean. Phys., 15, 67-71.

Thorpe, A. J., and C. H. Bishop, 1995: Potential vorticity and the electrostatic analogy: Ertel-Rossby formulation. Quart. J. Roy. Meteor. Soc., 121, 1477-1495.

Yaglom, A. M., and I. M. Yaglom, 1973: Probability and Information. 3d ed. Reidel, 512 pp. 